\title{
DINÂMICA EM SUPERFÍCIE, VOLUME, BIOMASSA E CARBONO NAS FLORESTAS NATIVAS BRASILEIRAS: 1990-2015
}

\author{
DYNAMICS OF FOREST COVER, VOLUME, BIOMASS, AND CARBON IN THE BRAZILIAN \\ NATIVE FORESTS: $1990-2015$
}

\author{
Carlos Roberto Sanquetta', Ana Paula Dalla Corte ${ }^{2}$, Allan Libanio Pelissari ${ }^{3}$, Margarida Tomé4, \\ Greyce Charllyne Benedet Maas ${ }^{5}$, Mateus Niroh Inoue Sanquetta ${ }^{6}$ \\ 1, 2, 3, 5, 6 Universidade Federal do Paraná, Curitiba, Paraná, Brasil - carlos_sanquetta@hotmail.com, \\ anapaulacorte@gmail.com,allanpelissari@gmail.com,greyce.maas@gmail.com \& \\ mateus.sanquetta@gmail.com
}

${ }^{4}$ Universidade de Lisboa, Instituto Superior de Agronomia, Lisboa, Portugal-magatome@ulisboa.pt

RESUMO

Avaliou-se a dinâmica em área e em estoques de volume de madeira, biomassa e carbono nas florestas nativas do Brasil no período 1990 a 2015. A fonte utilizada para o trabalho foi o Relatório FRA2015 (Avaliação dos Recursos Florestais) submetido pelo Serviço Florestal Brasileiro à FAO (Organização das Nações Unidas para Alimentação e Agricultura). Os dados publicados foram analisados criticamente, deduções dos parâmetros empregados foram realizadas e alguns ajustes nos cálculos foram efetivados. A área de florestas nativas no Brasil decresceu no período de 25 anos, de $542 \mathrm{M}$ ha para $486 \mathrm{M}$ ha, o que corresponde a $10 \%$ da superfície inicial computada em 1990 . As maiores perdas de áreas florestais ocorreram nos biomas Amazônia e Cerrado que representaram 85\% do total e 56 $\mathrm{M}$ ha em todo o País, sendo equivalente à superfície do Estado da Bahia. O estoque volumétrico de madeira foi reduzido em 8,45\% no período avaliado, de $103 \mathrm{G} \mathrm{m}^{3}$ para $95 \mathrm{G} \mathrm{m}^{3}$, com maior perda no bioma Amazônia (79\%). A biomassa total seca estocada nas florestas também decresceu 8,44\%, de $126 \mathrm{G}$ t para $115 \mathrm{G} \mathrm{t}$, com maior redução na Amazônia (79\%). O estoque de carbono caiu de $63 \mathrm{G} \mathrm{t}$ para $58 \mathrm{G} \mathrm{t}$, expressando uma perda de 8,40\% que se deu em maior intensidade no bioma Amazônia (80\%). Concluiu-se que as reduções em volume, biomassa e carbono são atribuídas à diminuição da cobertura florestal em todos os biomas e que tais reduções implicaram em emissões de gases de efeito estufa.

PALAVRAS-CHAVE: Biomas, Cobertura florestal, Desmatamento, Dióxido de carbono, Emissões.

\begin{abstract}
Dynamics of forest cover, wood volume, biomass, and carbon stocks in Brazilian native forests from 1990 to 2015 were examined. Data source was FRA2015 Report (Forest Resources Assessment) submitted by the Brazilian Forest Service to FAO (Food and Agriculture Organization of the United Nations). The published data were analyzed critically, deductions of the parameters utilized and some adjustments in the calculations were performed. The area of native forests decreased over a period of 25 years, from $542 \mathrm{M}$ ha to $486 \mathrm{M}$ ha, corresponding to $10 \%$ of the initial forest cover in 1990. The greatest loss of forests happened in the Amazon and Cerrado biomes, which represented $85 \%$ of the total loss, reaching $56 \mathrm{M}$ ha, equivalent to the Bahia State territory. Wood volume stock was reduced from 103 $\mathrm{Gm}^{3}$ to $95 \mathrm{G} \mathrm{m}^{3}$, or $8.45 \%$, with the highest loss in the Amazon biome (79\%). Total dry biomass stored in forests decreased from $126 \mathrm{G}$ t to $115 \mathrm{G} \mathrm{t}$, corresponding to $8.44 \%$, with the highest reduction also in the Amazon (79\%). Carbon stock diminished from $63 \mathrm{G}$ t to $58 \mathrm{G} \mathrm{t}$, a loss of $8.40 \%$, which was more remarkably noticed in the Amazon biome (80\%). It was concluded that the reductions in volume, biomass, and carbon are attributed to the reduction of forest cover in all biomes and that such reductions observed imply greenhouse gas emissions.
\end{abstract}

KEYWORDS: Biomes, Forest cover, Deforestation, Carbon dioxide, Emissions. 


\section{INTRODUÇÃO}

O Brasil possui a segunda maior área florestal em nível mundial, atrás apenas da Federação Russa. Esses dois países detêm mais de um quarto de todas as florestas do Globo (FAO, 2015).

Entre os países tropicais, o Brasil é o que abriga a maior extensão contínua de florestas, cujo ícone maior é a Amazônia. Os seis biomas brasileiros, oficialmente denominados Amazônia, Caatinga, Cerrado, Mata Atlântica, Pampa e Pantanal (IBGE, 2004), abrigam importantes recursos hídricos, expressiva biodiversidade e valiosas matérias primas.

Por outro lado, a perda de cobertura florestal do Brasil está entre as maiores do mundo (KEENAN et al., 2015), trazendo preocupações em diferentes âmbitos, apesar dos esforços empreendidos pelas autoridades em reduzir as taxas de desmatamento, sobretudo na Amazônia (ARIMA et al., 2016).

Para acompanhar o estado e as tendências da cobertura florestal, o Serviço Florestal Brasileiro coordena o Inventário Florestal Nacional (IFN) em andamento em todo o País (SFB, 2017). Apesar de ainda não ter concluído o seu inventário florestal, o Brasil é demandado continuamente pelas organizações internacionais a reportar suas estatísticas florestais, o que vem sendo realizado por intermédio de relatórios publicados periodicamente.

O Brasil aporta informações florestais à Organização das Nações Unidas para Alimentação e Agricultura - FAO (Food and Agriculture Organization of the United Nations) a cada cinco anos. Informa a dinâmica temporal das áreas florestais, os estoques volumétricos de madeira, biomassa e carbono, entre outros, no relatório intitulado "Avaliação dos Recursos Florestais", conhecido como FRA (Forest Resources Assessment em inglês) (FAO, 2014). O mais recente até o presente é o FRA2015.

$\mathrm{Na}$ elaboração do FRA, são utilizadas informações secundárias e indiretas disponíveis, relatórios oficiais e publicações. Ainda não há dados primários de todo o País que permitam nortear o FRA do Brasil, mas espera-se que com o avanço do IFN as novas edições do FRA sejam mais detalhadas, completas e precisas.

Apesar de ser um relatório oficial sobre o estado das florestas no Brasil, o FRA é pouco conhecido e divulgado no setor. As ricas informações nele contidas também não são alvo de análises críticas que possam auxiliar na sua melhoria. Além disso, há a ausência de um alinhamento do FRA do Brasil com outros relatórios nacionais correlatos, como o Inventário de Gases de Efeito Estufa (GEE) contido nas chamadas "Comunicações Nacionais" (MCTI, 2016) à Convenção Quadro das Nações Unidas sobre Mudanças Climáticas (UNFCCC United Nations Framework Convention on Climate Change).

A hipótese abordada é que as florestas nativas estocam grande quantidade de carbono, porém, a perda de cobertura florestal no período considerado, reduz esse estoque e implica em emissões de GEE.

O presente trabalho tem como objetivo examinar a dinâmica das áreas florestais e dos estoques volumétricos de madeira, biomassa e carbono nas florestas nativas brasileiras no período 1990-2015, baseando-se nas informações reportadas no FRA2015, bem como efetuar uma análise crítica da sua metodologia e dos seus resultados.

\section{MATERIAL E MÉTODOS}

\section{Dados utilizados}

A fonte de dados utilizadas neste estudo é o relatório intitulado Global Forest Resources Assessment FRA2015, Brazil (FAO, 2014). Foram utilizados dados de áreas florestais, volumes de madeira e estoques de biomassa e carbono nos anos 1990, 2000, 2005, 2010 e 2015, em todos os seis biomas brasileiros. Ademais, foram utilizadas apenas os dados das áreas tipicamente florestais, desconsiderando-se outras formações ou tipologias de uso do solo, mesmo que com ocorrência de árvores (other wooded land).

\section{Dedução dos parâmetros empregados no FRA2015}

Para melhor clarificar os critérios de elaboração dos FRA015, foram feitas deduções de alguns parâmetros importantes na conversão das áreas florestais para volume, biomassa e carbono ao longo do tempo.

Conforme definição metodológica do FRA, "Volume consiste do volume total do fuste com casca de todas as árvores vivas com um diâmetro mínimo de $10 \mathrm{~cm}$ na altura de 1,30m (ou acima das imperfeições anotando-se a altura da medição), excluindo-se os galhos". Para cada bioma foi deduzido um valor médio representativo por bioma.

Os parâmetros examinados foram os estoques volumétricos, de biomassa e carbono por hectare para cada bioma, a razão de raízes $(R)$ para conversão da biomassa acima do solo (aérea) para biomassa total; o fator de conversão e expansão da biomassa (FCEB), que converte volume para biomassa total; e o teor de carbono (TC) que converte biomassa em estoque de carbono.

Para obter os valores por hectare das variáveis volume, biomassa e carbono, os valores totais por bioma foram divididos pelas respectivas áreas florestais contidas no FRA0215. O valor $R$ foi obtido pela divisão da biomassa abaixo 
do solo pela biomassa acima do solo. O FCEB foi calculado pela divisão da biomassa total pelo volume, enquanto o $T C$ foi determinado pela divisão da biomassa pelo respectivo estoque de carbono.

\section{Correção nos estoques de carbono}

Em virtude de um possível equívoco detectado na quantificação de carbono no FRA2015 na aplicação do teor de carbono no bioma Mata Atlântica, foi necessário proceder à correção dos valores apresentados no relatório. 0 valor 0,74 foi empregado erroneamente no FRA2015, quando o correto seria 0,47 , sendo considerado o valor padrão sugerido pelo Painel Intergovernamental sobre Mudanças Climáticas - IPCC (IPCC, 2006).

\section{Emissões de dióxido de carbono}

Foram calculadas as emissões em dióxido de carbono equivalente correspondentes à mudança no estoque de carbono entre os anos 1990 e 2015, assumindo oxidação total e instantânea. Para tanto, a diferença nos estoques de carbono no período foi multiplicada por 44/12, valor da conversão estequiométrica da massa do elemento $\mathrm{C}$ para a molécula de $\mathrm{CO}_{2}$, em que $\mathrm{C}=12, \mathrm{O}=16 \mathrm{e}$, portanto, $\mathrm{CO}_{2}=44$.

\section{RESULTADOS E DISCUSSÃO}

\section{Cobertura florestal}

O período de 25 anos considerado neste estudo marca uma expressiva redução na superfície florestal do País em todos os biomas. As florestas representavam 63,61\% da superfície oficial do Brasil (IBGE, 2004) em 1990 e passaram a $57,05 \%$ em 2015. A redução de cobertura florestal implicou na perda de 55.918.786 ha de vegetação arbórea nativa, o que corresponde a $10,32 \%$ da área inicialmente computada em 1990. Essa perda correspondeu a aproximadamente à área do Estado da Bahia. O período de maior perda de florestas foi entre 2000 e 2005, com aproximadamente $3 \mathrm{M}$ ha por ano, em média.

Todos os biomas tiveram redução de área florestal, mas, por outro lado, também foi observada a diminuição da taxa de desflorestamento em todos os biomas a partir do ano de 2005. A maior perda absoluta de cobertura florestal ocorreu no bioma Amazônia, com cerca de $28 \mathrm{M}$ ha (que implicou em 7,52\% de redução no período 1990-2015), seguido do Cerrado, com $20 \mathrm{M}$ ha, que teve a perda maior em termos relativos a área do mesmo, com $22,36 \%$ de diminuição de sua cobertura (no mesmo período de período 1990-2015) (Tabela
1 no final do documento). No caso da Mata Atlântica a redução foi da ordem de 3,5\% no mesmo período.

A redução da cobertura florestal natural no mundo tem ocorrido como resultado, principalmente, da derrubada de árvores para fins madeireiros, devastação de terras para utilização da agropecuária, incêndios e fenômenos naturais (ARRAES et al., 2012). No Brasil atual, a maior parte da perda de florestas, notadamente na Amazônia, se deve à expansão da fronteira agropecuária, abertura de estradas, extração de madeira e construção de hidrelétricas. A pecuária, particularmente, é considerada o principal vetor de desmatamento (COHN et al., 2014; STEINWEG et al., 2016).

\section{Dedução dos parâmetros utilizados no FRA2015}

Os estoques de volume, biomassa e carbono reportados pelo FRA2015 advêm da multiplicação das áreas florestais (Tabela 1) por valores unitários (por hectare), que, embora não tenham sido reportados no referido relatório, puderam ser deduzidos neste trabalho (Tabela 2).

Tabela 2. Valores de volume, biomassa e carbono por hectare, FCEB, $R$ e TC deduzidos a partir do FRA2015

\begin{tabular}{ccccccc}
\hline Bioma & $\begin{array}{c}\text { Volume } \\
\left(\mathbf{m}^{3} \mathbf{h a}^{-1}\right)\end{array}$ & $\begin{array}{c}\text { Biomassa } \\
\left(\mathrm{t} \mathrm{ha}^{-1}\right)\end{array}$ & $\begin{array}{c}\text { Carbono } \\
\left(\mathrm{t} \mathrm{ha}^{-1}\right)\end{array}$ & $\begin{array}{c}\text { FCEB } \\
\left(\mathrm{t} \mathrm{t}^{-1}\right)\end{array}$ & $\begin{array}{c}\boldsymbol{R} \\
\left(\mathrm{t} \mathrm{t}^{-1}\right)\end{array}$ & $\begin{array}{c}\boldsymbol{T C} \\
\left(\mathrm{t} \mathrm{t}^{-1}\right)\end{array}$ \\
\hline Amazônia & 247 & 303 & 152 & 1,22 & 0,19 & 0,50 \\
\hline Caatinga & 63 & 63 & 30 & 1,00 & 0,27 & 0,47 \\
$\begin{array}{c}\text { Cerrado } \\
\text { Mata }\end{array}$ & 66 & 83 & 39 & 1,25 & 0,59 & 0,48 \\
Atlântica & 97 & 129 & 61 & 1,33 & 0,22 & $0,74^{*}$ \\
Pampa & 35 & 59 & 28 & 1,69 & 0,23 & 0,47 \\
\hline Pantanal & 74 & 80 & 38 & 1,07 & 0,48 & 0,48 \\
\hline Geral & 191 & 233 & 116 & 1,22 & 0,22 & 0,50 \\
\hline
\end{tabular}

* Valor desconsiderado neste estudo, sendo substituído por 0,47 (default do IPCC). FCEB: fator de conversão e expansão de biomassa. $R$ : razão de raízes. $T C$ : teor de carbono.

Os valores da variável volume (Tabela 2) foram obtidos por compilação a partir de várias literaturas utilizadas e citadas no FRA2015 o qual foi tomado como base (FAO, 2014), o mesmo ocorrendo para biomassa e carbono. É importante também destacar que o FRA2015 não mostra modificação nos valores unitários por hectare dessas variáveis ao logo do tempo, permanecendo inalterados nos 25 anos analisados.

Os estoques volumétricos por hectare variaram de acordo com o bioma. O maior volume foi verificado na Amazônia e o menor volume no Pampa. O mesmo comportamento se observa para biomassa e carbono. 
Tabela 1. Síntese dos valores de área florestal, volume de madeira, biomassa e carbono nas florestas nativas do Brasil, por bioma, entre os anos 1990 e 2015

\begin{tabular}{|c|c|c|c|c|c|c|c|}
\hline Bioma & Variável & Unidade & 1990 & 2000 & 2005 & 2010 & 2015 \\
\hline \multirow{4}{*}{ Amazônia } & Área & hectare & 369.820 .791 & 357.222 .758 & 348.750 .559 & 344.421 .391 & 342.027 .340 \\
\hline & Volume & $\mathrm{M} \mathrm{m}^{3}$ & 91.492 & 88.375 & 86.279 & 85.208 & 84.616 \\
\hline & Biomassa & $\mathrm{Mt}$ & 111.938 & 108.124 & 105.560 & 104.250 & 103.525 \\
\hline & Carbono & $\mathrm{Mt}$ & 56.112 & 54.201 & 52.915 & 52.259 & 51.895 \\
\hline \multirow{4}{*}{ Caatinga } & Área & hectare & 46.490 .458 & 43.906 .894 & 42.615 .112 & 41.480 .794 & 40.582 .671 \\
\hline & Volume & $\mathrm{M} \mathrm{m}^{3}$ & 2.937 & 2.774 & 2.692 & 2.621 & 2.564 \\
\hline & Biomassa & $\mathrm{Mt}$ & 2.931 & 2.768 & 2.686 & 2.615 & 2.558 \\
\hline & Carbono & $\mathrm{Mt}$ & 1.390 & 1.313 & 1.275 & 1.241 & 1.214 \\
\hline \multirow{4}{*}{ Cerrado } & Área & hectare & 89.175 .265 & 79.803 .561 & 75.117 .709 & 71.373 .852 & 69.235 .988 \\
\hline & Volume & $\mathrm{M} \mathrm{m}^{3}$ & 5.885 & 5.266 & 4.957 & 4.710 & 4.569 \\
\hline & Biomassa & $\mathrm{Mt}$ & 7.380 & 6.605 & 6.217 & 5.907 & 5.730 \\
\hline & Carbono & $\mathrm{Mt}$ & 3.515 & 3.145 & 2.961 & 2.813 & 2.729 \\
\hline \multirow{4}{*}{ Mata Atlântica } & Área & hectare & 22.579 .479 & 22.208 .350 & 22.022 .785 & 21.871 .166 & 21.770 .466 \\
\hline & Volume & $\mathrm{M} \mathrm{m}^{3}$ & 2.189 & 2.153 & 2.135 & 2.120 & 2.111 \\
\hline & Biomassa & $\mathrm{Mt}$ & 2.908 & 2.860 & 2.836 & 2.816 & 2.803 \\
\hline & Carbono & $\mathrm{Mt}$ & 1.367 & 1.344 & 1.333 & 1.324 & 1.318 \\
\hline \multirow{4}{*}{ Pampa } & Área & hectare & 3.663 .163 & 3.477 .510 & 3.384 .683 & 3.295 .129 & 3.210 .486 \\
\hline & Volume & $\mathrm{M} \mathrm{m}^{3}$ & 127 & 120 & 117 & 114 & 111 \\
\hline & Biomassa & $\mathrm{Mt}$ & 215 & 204 & 199 & 193 & 188 \\
\hline & Carbono & $\mathrm{Mt}$ & 101 & 96 & 93 & 91 & 89 \\
\hline \multirow{4}{*}{ Pantanal } & Área & hectare & 9.991 .603 & 9.479 .361 & 9.223 .240 & 9.042 .555 & 8.975 .022 \\
\hline & Volume & $\mathrm{M} \mathrm{m}^{3}$ & 744 & 706 & 687 & 673 & 668 \\
\hline & Biomassa & $\mathrm{Mt}$ & 795 & 754 & 734 & 719 & 714 \\
\hline & Carbono & $\mathrm{Mt}$ & 381 & 361 & 352 & 345 & 342 \\
\hline \multirow{4}{*}{ Total } & Área & hectare & 541.720 .759 & 516.098 .434 & 501.114 .088 & 491.484 .887 & 485.801 .973 \\
\hline & Volume & $\mathrm{M} \mathrm{m}^{3}$ & 103.373 & 99.395 & 96.868 & 95.447 & 94.639 \\
\hline & Biomassa & $\mathrm{Mt}$ & 126.166 & 121.315 & 118.231 & 116.500 & 115.519 \\
\hline & Carbono & $\mathrm{Mt}$ & 62.866 & 60.461 & 58.928 & 58.071 & 57.586 \\
\hline
\end{tabular}

*Adaptado de FAO (2014)

Os valores de FCEB variaram entre biomas. Os FCEB deduzidos a partir do FRA 2015 foram superiores aos valores padrões sugeridos pelo IPCC (IPCC, 2006), exceto no bioma Amazônia. Um valor de FCEB maior implica maior quociente da razão entre a biomassa total e o volume, sendo dependente da arquitetura das árvores e da densidade dos tecidos vegetais, sobretudo do lenho. O fato de o FRA se apoiar em publicações sobre os biomas brasileiros, ele confere maior confiabilidade às estimativas. Contudo, evidencia-se a necessidade de se desenvolver mais pesquisas orientadas à obtenção dessa grandeza, bem como a sistematização de valores para os diferentes biomas do País. Sem dúvida, esse tipo de estudo poderá contribuir muito para melhorar a qualidade das informações a serem reportados em futuras edições do FRA. O FCEB é particularmente importante nesse contexto, pois permite efetuar a conversão do volume comercial de madeira calculado nos inventários florestais usuais em biomassa e carbono (NOGUEIRA et al., 2008; SILVEIRA, 2010).

O valor de $R$, que indica a proporção de raízes na biomassa, também é diferente para cada bioma. Para a Amazônia e a Mata Atlântica, os valores apresentados são muito inferiores ao padrão de 0,37 do IPCC para florestas tropicais úmidas. O valor para a Caatinga está de acordo com o sugerido pelo IPCC para florestas tropicais secas, de 0,27 a 0,28 . No caso do Cerrado, o valor adotado no FRA é superior 
a todos os sugeridos pelo IPCC, mas salienta-se que não há especificação daquela instituição para savanas tropicais. Não existe também um padrão recomendado para a tipologia do Pampa. Para o Pantanal, o valor de $R$ é superior aos valores apresentados pelo IPCC para tipologias similares.

É importante destacar que o valor $R$ é importante nas estimativas de biomassa e carbono, uma vez que repercute nos estoques abaixo do solo (subterrâneos) e no total (acima e abaixo do solo). Qualquer alteração na relação expressa por $R$ afeta todas as estimativas (PETRESCU et al., 2008). Portanto, quando do uso dessa variável na estimação de biomassa e carbono, é indispensável fazer um exame minucioso da literatura e decidir pela aplicação de um valor conservador e realista. Dessa forma, evidencia-se a importância de se incentivar pesquisas que forneçam valores de $R$ para os diferentes biomas e formações vegetacionais, aplicando-se metodologia alinhada com os guias do IPCC. Vale dizer que publicações existem, mas essas nem sempre são padronizadas, notadamente no que tange à profundidade de escavação e ao tamanho de inclusão das raízes.

Os valores de TC aplicados no FRA2015 também variam caso a caso, dependendo do bioma. Uma vez que os valores padrões do IPCC para florestas nos trópicos variam de 0,43 a 0,49 , é pertinente afirmar que, de modo geral, os valores utilizados no relatório à $\mathrm{FAO}$ estão de acordo. A única exceção é a Mata Atlântica, cujo TC deduzido é de 0,74, valor esse considerado anormal. Provavelmente ocorreu engano na aplicação desse valor, tendo havido troca de 0,47 por 0,74. Em virtude disso, o valor de TC nesse bioma foi reconsiderado e o estoque de carbono recalculado. Esse parâmetro também tem implicações importantes na quantificação de carbono (THOMAS \& MARTIN, 2012).

\section{Estoques volumétricos, de biomassa e carbono}

O estoque volumétrico decresceu $8,45 \%$, de $103 \mathrm{G} \mathrm{m}^{3}$ em 1990 para $95 \mathrm{G} \mathrm{m}^{3}$ em 2015. O bioma Amazônia foi responsável por $78,71 \%$ dessa redução, o qual perdeu 7,52\% do seu estoque. Porém, a maior perda relativa de volume deu-se no bioma Cerrado, que teve uma redução de $22,36 \%$ em 25 anos.

A biomassa estocada nas florestas nativas brasileiras, em virtude da perda de cobertura florestal, foi reduzida em $8,44 \%$ nos 25 anos, de $126 \mathrm{G}$ t para $115 \mathrm{G}$ t. Novamente, a maior perda deu-se no bioma Amazônia, que foi responsável por $79,01 \%$ dessa redução, correspondendo a 7,52\% de sua biomassa estocada. A maior perda relativa foi verificada no Cerrado, com 22,36\%.

A redução no estoque de carbono foi de $5.280 \mathrm{M}$ t no período, de 63 G t em 1990 para 58 G t em 2015. Isso corresponde a 8,40\% do estoque inicial computado em 1990. A perda de estoque de carbono nos biomas seguiu o mesmo comportamento da biomassa, uma vez que são variáveis diretamente relacionadas.

\section{Emissões de dióxido de carbono}

A perda de estoque de carbono nas florestas nativas brasileiras no período 1990 a 2015 foi de 5,28 G t. Considerando oxidação total e instantânea, essa perda correspondeu à emissão de 19,36 G t CO 2eq. $_{\text {, aplicando-se as }}$ respectivas massas atômicas.

O bioma Amazônia é responsável por 79,87\% dessas emissões e o Cerrado por $14,88 \%$, com os demais biomas participando com pouco mais de $5 \%$. Essa emissão bruta estimada é consideravelmente menor que os quantitativos divulgados na III Comunicação Nacional - Inventário Brasileiro de Gases de Efeito Estufa (MCTI, 2016) e que o Sistema de Emissão de Gases de Efeito Estufa - SEEG (OBSERVATÓRIO DO CLIMA, 2018).

Considerando que a redução no estoque de carbono no mundo foi de 13,6 G t entre 1990 e 2015 (KÖHL et al., 2015), ou seja, 49,88 G t CO 2 eq., o Brasil foi responsável por 38,82\% das emissões globais por perda de cobertura florestal no período considerado.

\section{CONCLUSÕES}

Em 25 anos, houve perda de 10\% na cobertura florestal do País, correspondente à área do Estado da Bahia;

O bioma Amazônia foi o que perdeu a maior área florestal, seguida do Cerrado, que teve a maior perda relativa de cobertura;

Os estoques de volume, biomassa e carbono decresceram em todos os biomas no período de análise, em decorrência da perda de cobertura florestal;

A perda de estoque de carbono nas florestas nativas brasileiras no período 1990 a 2015 implicou na emissão de grandes quantidades de dióxido de carbono para a atmosfera. A emissão do Brasil por perda de cobertura florestal nesse período representa $38,82 \%$ das emissões de GEE globais.

\section{REFERÊNCIAS}

ARIMA, E.Y. et al. Public policies can reduce tropical deforestation: lessons and challenges from Brazil. Land Use Policy, v.41, p.465-473, 2016.

ARRAES, R.A. et al. Causas do Desmatamento no Brasil e seu Ordenamento no Contexto Mundial. Revista de Economia e Sociologia Rural, v.50, n.1, p.119-140, 2012. 
COHN, A.S. et al. Cattle ranching intensification in Brazil can reduce global greenhouse gas emissions by sparing land from deforestation. Proceedings of the National Academy of Sciences, v.111, n.20, p.7236-7241, 2014.

FAO - Food and Agriculture Organization of the United Nations. Global Forest Resources Assessment 2015. FAO Forestry Paper No. 1. Rome: 2015.

FAO - Food and Agriculture Organization of the United Nations. FRA2015 Brazil, Country Report. Rome: FAO, 2014. Disponível em: http://www.fao.org/3/a-az172e.pdf

IBGE - INSTITUTO BRASILEIRO DE GEOGRAFIA E ESTATÍSTICA. Mapa de Biomas e de Vegetação, 2004. Disponível em: https://ww2.ibge.gov.br/home/presidencia/noticias/21052004bio mashtml.shtm

IPCC - INTEGOVERNMENTAL PANEL ON CLIMATE CHANGE. 2006 IPCC Guidelines for National Greenhouse Gas Inventories, Volume 4. Disponível em: http://www.ipcc-nggip.iges.or.jp/public/ 2006gl/pdf/4 Volume4/V4 04 Ch4 Forest Land.pdf

KEENAN, R.J. et al. Dynamics of global forest area: Results from the FAO Global Forest Resources Assessment 2015. Forest Ecology and Management, v.352, n.7, p.9-20, 2015.

KÖHL, M. et al. Changes in forest production, biomass and carbon: Results from the 2015 UN FAO Global Forest Resource Assessment. Forest Ecology and Management, v.352, n.7, p.21-34, 2015.

MCTI - MINISTÉRIO DA CIÊNCIA, TECNOLOGIA E INOVAÇÃO. III Comunicação Nacional do Brasil à Convenção-Quadro das Nações Unidas sobre Mudança do Clima. Volume III. Brasília: 2016.

NOGUEIRA, E.M. et al. Estimates of forest biomass in the Brazilian Amazon: new allometric equations and adjustments to biomass from wood-volume inventories. Forest Ecology and Management, v.256, p.1853-1867, 2008.

OBSERVATÓRIO DO CLIMA. Sistema de estimativas de emissões e remoções de gases de efeito estufa (SEEG), 2018. Disponível em: http://plataforma.seeg.eco.br/sectors/mudanca-de-uso-da-terra-efloresta

PETRESCU, A.M.R. et al. Global estimates of carbon stock changes in living forest biomass: EDGARv4.3 - time series from 1990 to 2010. Biogeosciences, v.9, p.3437-3447, 2012.

SERVIÇO FLORESTAL BRASILEIRO (SFB). O que é o Inventário Florestal Nacional. Brasília, 2017. Disponível em: http://www.florestal.gov.br/inventario-florestal-nacional

SILVEIRA, P. Estimativa da biomassa e carbono acima do solo em um fragmento de Floresta Ombrófila Densa utilizando o método da derivação do volume comercial. Floresta, v.40, n.4, p.789-800, 2010.

STEINWEG, T. et al. Economic drivers of deforestation: sector exposed to sustainability and financial risks. Washington: Chain Reaction Research, 2016 Disponivel em: http://www.aidenvironment.org/wp-content/uploads/2016/08/ economic-drivers-of-deforestation-crr-160803-final.pdf
THOMAS, S.C.; MARTIN, A.R. Carbon content of tree tissues: a synthesis. Forests, v.3, n.2, p.332-352, 2012. 